\title{
The SASTRA Ramanujan Prize: Its Origins and Its Winners
}

\section{Krishnaswami Alladi}

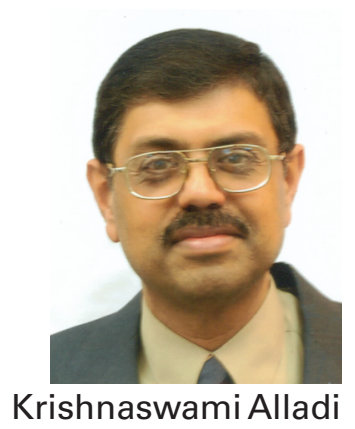

The SASTRA Ramanujan Prize is a $\$ 10,000$ annual award given to mathematicians not exceeding the age of 32 for path-breaking contributions in areas influenced by the genius Srinivasa Ramanujan. The prize has been unusually effective in recognizing at an early stage in their careers extremely gifted mathematicians who have gone on to accomplish even greater things in mathematics and be awarded prizes with a hallowed tradition such as the Fields Medal. This is due to the enthusiastic support from leading mathematicians around the world and the caliber of the winners. The age limit of 32 is because Ramanujan lived only for 32 years, and in that brief lifespan made revolutionary contributions; so the challenge for the prize candidates is to show what they have achieved in that same time frame! The way the prize was conceived and launched is an incredible story, which I will relate here. I will then briefly describe some major aspects of the work of the winners.

\section{The Origins}

The district of Tanjore (=Tanjavur) in the state of Tamil Nadu in South India has been a seat of culture for several centuries. Tanjore has produced some of greatest composers and performers of South Indian classical music. Tanjore is well known for art in various forms and has a great concentration of Hindu temples. It is in this region steeped in culture that Ramanujan was born on December 22, 1887, in the town of Kumbakonam, where he lived until he completed high school.

Krishnaswami Alladi is professor of mathematics at the University of Florida, Gainesville. His email address is a11adi k@uf1.edu.

For permission to reprint this article, please contact: reprint -permission@ams.org.

DOI: https://dx.doi.org/10.1090/noti 1765
During the second half of the twentieth century, Ramanujan's humble home in Kumbakonam, from where a thousand theorems emerged, was in a dilapidated condition. Even though in India many events and programs were held regularly in Ramanujan's memory, including the grand Ramanujan Centenary celebrations in December 1987, nothing was done for the renovation of this historic home. One of the most significant developments in the worldwide effort to preserve and honor the legacy of Ramanujan is the purchase in 2003 of Ramanujan's home in Kumbakonam by SASTRA University to maintain it as a museum. This purchase had far-reaching consequences because it led to the involvement of a university in the preservation of Ramanujan's legacy for posterity.

The Shanmugha Arts, Science, Technology and Research Academy (SASTRA), is a private university in the town of Tanjore after which the district is named. SASTRA was founded in 1984 and has grown by leaps and bounds. Admission is very competitive, and SASTRA has succeeded in attracting some of the brightest students in India.

SASTRA has renovated Ramanujan's home beautifully without altering its structure or design. The only modification was to add a bust of Ramanujan in the living room. In connection with the purchase of Ramanujan's home, SASTRA University opened a branch campus in Kumbakonam in 2003, called the Srinivasa Ramanujan Centre. SASTRA has a museum there in which several important letters, photographs, and documents related to Ramanujan are displayed. A visit to Kumbakonam just to see Ramanujan's home and this museum will be worthwhile and inspiring.

One afternoon in September 2003, I received a phone call from S. Swaminathan, then a graduate student at the University of Virginia (now a dean at SASTRA). He introduced himself as the son of Vice-Chancellor R. Sethur- 
aman of SASTRA University, gave me some background about SASTRA, and said that to mark the occasion of the purchase of Ramanujan's home, SASTRA would be conducting an international conference that year in December at the Kumbakonam campus. The President of India, Dr. Abdul Kalam, had agreed to inaugurate this conference on December 20, 2003 and declare Ramanujan's home as a national treasure. Swaminathan invited me to bring a team of mathematicians from abroad to the conference, and said that SASTRA would cover all their expenses including international travel. I felt that what I heard over the phone was incredible because international airfare is rarely paid for mathematical conferences in India. But on checking I realized that something important was taking place. So I called George Andrews of Pennsylvania State University, the world's foremost authority in the theory of partitions and on Ramanujan's work, told him about SASTRA, and requested that he come to the conference. He said that, based on my assurance, he would come. In fact Andrews gave the opening lecture of the conference as well as the concluding Ramanujan Commemoration Lecture on December 22, Ramanujan's birthday. At the valedictory function, the participants suggested that SASTRA should conduct conferences annually around Ramanujan's birthday on different areas of mathematics influenced by Ramanujan. Dean Vaidhyasubramaniam of SASTRA agreed to this suggestion and invited me to help organize these annual conferences. I have done this annually since 2003, and so have also had the pleasure of being in Ramanujan's hometown each year in December thus making it an annual pilgrimage for me (see article 25 in [1], pp. 153-160).

For the second SASTRA Ramanujan Conference in 2004, the Vice-Chancellor invited me to inaugurate it and also deliver the concluding Ramanujan Commemoration lec-

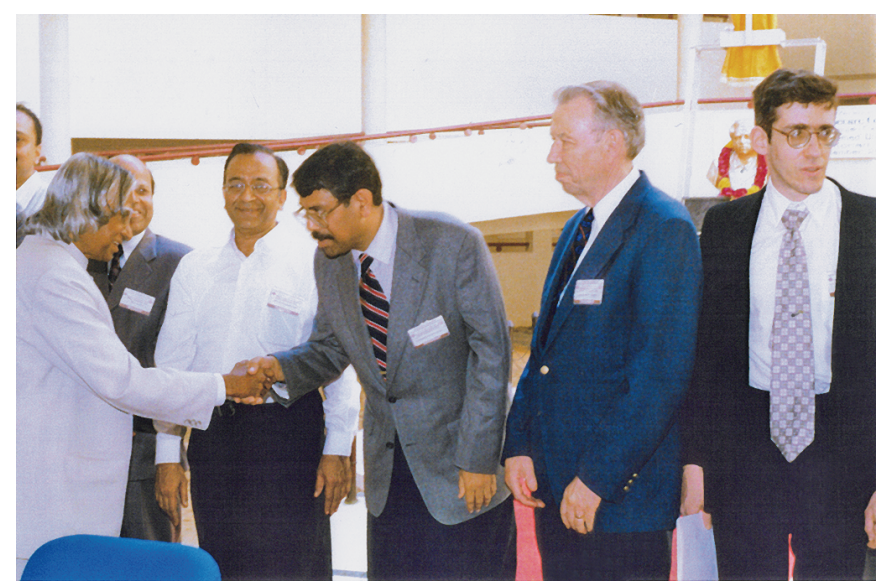

Figure 1. Krishna Alladi being introduced to the President of India, Dr. Abdul Kalam, during the First Ramanujan Conference at SASTRA University on December 20, 2003. To the left of Krishna are George Andrews (Penn State) and Noam Elkies (Harvard). ture. I was honored to get such an invitation, and I accepted it happily.

Conference inaugurations in India are usually elaborate and grand ceremonies starting with the traditional "lighting of the lamp" by the Chief Guest, with the lamp representing enlightenment through knowledge. There was a large gathering in the auditorium, and as we walked up to the stage to be seated before the official start of the ceremony, the Vice-Chancellor whispered to me and said, "I wish to give $\$ 10,000$ annually for a worthy cause in the name of Ramanujan. You please decide how it should be used and announce it in your inaugural speech." I was pleasantly shocked by this and said, "This is very generous of you, but are you asking me to announce this in the next few minutes without consulting anybody?" He said, "Yes, consulting people, or going through a Committee, will cause unnecessary delays, and so I want you to come to decision right now and announce it." Fortunately, my inaugural speech was preceded by the lamp lighting ceremony and the customary elaborate welcome of the audience and the introduction of the Chief Guest (me) as is common in India, and so I had at least fifteen minutes to think about it as all this was going on! When I got up to speak, I mentioned this very generous offer from the Vice-Chancellor, and suggested that a SASTRA Ramanujan Prize be created and that $\$ 10,000$ be given to a mathematician not exceeding the age of 32 for outstanding contributions to areas influenced by Ramanujan. I pointed out that the Fields Medal is given only to those under 40 years of age, and the SASTRA Prize would be given to even younger mathematicians, some of whom may later win the Fields Medal or other well-established major prizes. The Vice-Chancellor liked this suggestion very much, and at the conclusion of the inaugural ceremony, in thanking me, he confirmed that the prize would be launched the next year. He then turned towards me and said, "I request you to be the Chair of the Prize Committee." So that is how the SASTRA Ramanujan Prize was conceived and launched, and how I got involved with the Prize. All this happened within the span of an hour during the inauguration.

Upon return to the United States, I called George Andrews and told him this incredible story of the launch of the prize and said that for this to be a success, I would need the support of the mathematical community. In particular, I wanted his presence on the Committee for the first year. He was surprised and happy to hear this and agreed to serve on the Prize Committee. Over the years, eminent mathematicians have supported the prize by either serving on the Prize Committee, making nominations, or writing letters evaluating the work of the nominees. The prize is given annually at SASTRA University, Kumbakonam, during an international conference held around Ramanujan's birthday. 


\section{COMMUNICATION}

\section{The winners}

The 2005 Prizes: Manjul Bhargava of Princeton University and Kannan Soundararajan of the University of Michigan came out on top as the strongest young mathematicians in the areas of algebraic number theory and analytic number theory, respectively, and were equally ranked 1 by the Committee.

One of the pioneering discoveries of Gauss was the composition law for binary quadratic forms. Introducing several new and unexpected ideas, Bhargava broke an impasse since the time of Gauss, and established in his $2001 \mathrm{PhD}$ thesis at Princeton University composition laws for higher degree forms. Bhargava applied these to solve new cases of one of the fundamental questions of number theory, that of the asymptotic enumeration of number fields of a given degree. He published the results in his thesis in a series of papers in the Annals of Mathematics. Bhargava's lecture at SASTRA University upon accepting the prize was on a different topic; he announced his joint work with Jonathan Hanke on the solution of the problem of determining all universal quadratic forms-a problem whose origin can be traced back to Ramanujan.

Kannan Soundararajan had made spectacular contributions to analytic number theory, most notably pertaining to the Riemann zeta function and Dirichlet $L$-functionsespecially on the distribution and location of their zeros. He also established deep results in random matrix theory, which has fundamental connections with prime number theory. In his PhD thesis at Princeton University, he showed that 7/8-ths of the quadratic $L$-functions have no zeros at

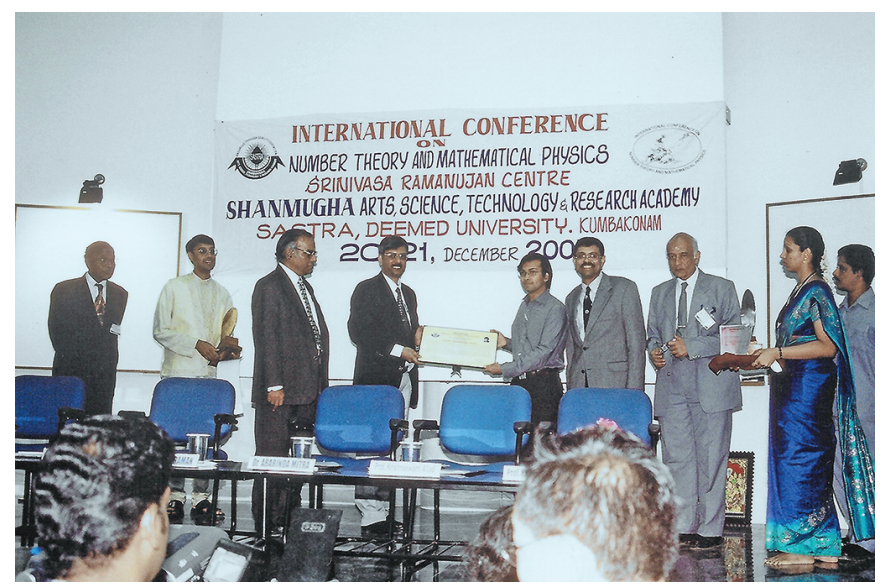

Figure 2. Kannan Soundararajan (then at Michigan) receiving the first SASTRA Ramanujan Prize on December 20, 2005 from Dr. Arabinda Mitra, Director, Department of Science and Technology, India. (Second from Left) Manjul Bhargava (Princeton) who had received the same prize a few minutes earlier, looks on. Next to Mitra is SASTRA Vice-Chancellor R. Sethuraman, and next to Soundararajan is Krishna Alladi. the critical point $s=1 / 2$, which provided strong evidence for a certain conjecture of Chowla. Along with Brian Conrey, he had shown that a positive proportion of Dirichlet $L$-functions have no zeros on the real axis within the critical strip.

The Prize Committee felt that both candidates deserved the full award. The SASTRA Vice-Chancellor generously agreed to the recommendation of the Committee that Bhargava and Soundararajan be awarded two full prizes, and that it should not be split. Thus the SASTRA Ramanujan Prize could not have had a better start (see Ken Ono's article [3] on the award of the first SASTRA Ramanujan Prizes, and mine ([1], p. 161-166) as well).

Bhargava continued producing fundamental work after receiving the SASTRA Ramanujan Prize, especially related to the average rank of elliptic curves. He was recognized with the AMS Cole Prize in 2008 and the Fields Medal in 2014.

When Soundararajan received the SASTRA Prize, he was a tenured faculty member at Michigan, and in the next year, was appointed full professor at Stanford. Subsequently, he was recognized with the Infosys Prize and the Ostrowski Prize, both in 2011.

The 2006 Prize: Thirty-one-year-old Terence Tao of UCLA had made far-reaching contributions to diverse areas of mathematics such as number theory, harmonic analysis, partial differential equations, and ergodic theory. He was widely regarded as one of the most influential mathematicians of our time.

One of Tao's most notable contributions was for the Kakeya Problem in higher dimensions. One aspect of the problem is to determine the fractal dimension of a set obtained by rotating a needle in $n$-dimensional space. In joint work with Nets Katz, Isabella Laba, and others, he improved all previously known estimates for the fractal dimension with ingenious combinatorial ideas. Another of Tao's seminal contributions was his joint work with Ben Green on long arithmetic progressions of prime numbers. One of the deepest results in this area is due to the Hungarian mathematician Szemerédi who showed that any set of positive density will have arbitrarily long arithmetic progressions. Subsequently Tim Gowers of Cambridge University gave a very different proof of Szemerédi's theorem. This result of Szemerédi does not apply to the set of primes, which is of zero density. By combining the ideas of Gowers along with tools from ergodic theory, Green and Tao proved the sensational result that there are arbitrarily long arithmetic progressions of primes.

Yet another fundamental contribution of Tao concerns the sum-product problem due to Paul Erdős and Szemerédi. Roughly speaking, this problem states that either the sum set or the product set of a set of $N$ numbers must be large. Tao was the first to recognize the significance of this problem in combinatorial number theory and harmonic analysis. In collaboration with Nets Katz and Jean 


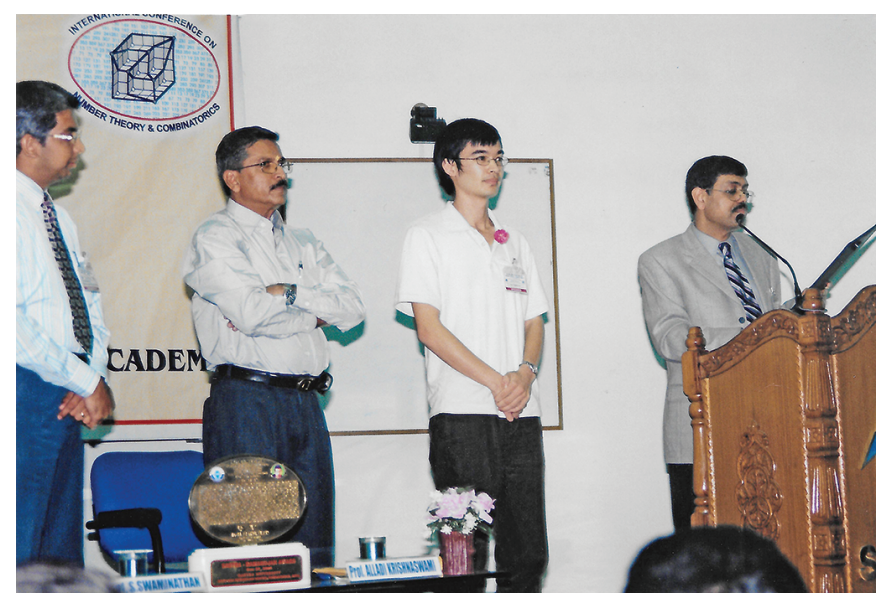

Figure 3. Krishna Alladi reads the citation before the 2006 SASTRA Ramanujan Prize is presented to TerenceTao (standing, middle) of UCLA.

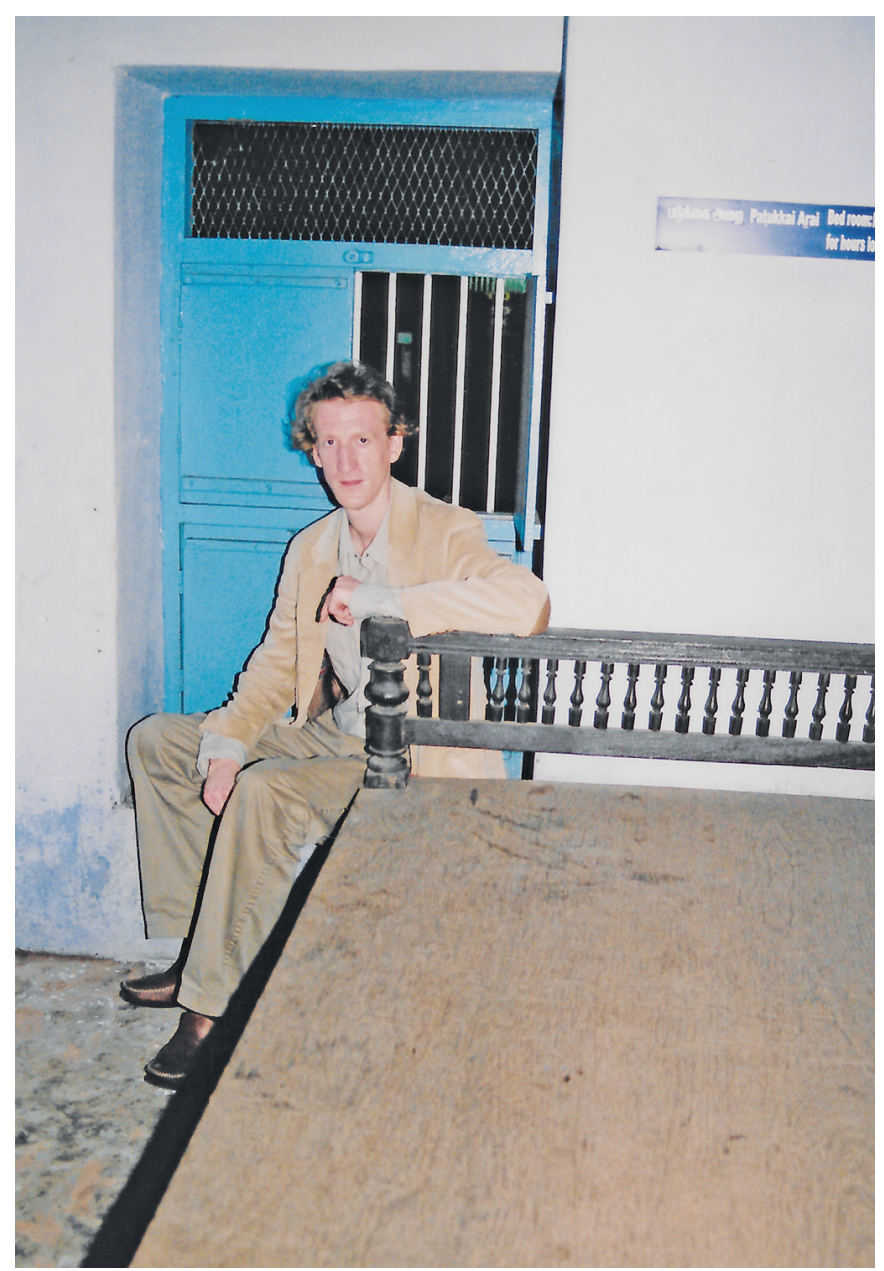

Figure 4. 2007 SASTRA Prize Winner Ben Green (then at Cambridge University) seated on the windowsill of the bedroom at Ramanujan's home. The cot there was the only one for Ramanujan's family. As a boy, Ramanujan used to sit on the windowsill and do his "sums" watching the passers-by on the street.
Bourgain, Tao made important generalizations that led to breakthroughs in harmonic analysis and number theory.

Honors have come to him in a steady stream. In 2006 Tao was awarded both the Fields Medal and the SASTRA Ramanujan Prize. More recently, he received the 2014 Breakthrough Prize. He currently holds the James and Carol Collins Chair at UCLA.

The 2007 Prize: Ben Green of Cambridge University had made phenomenal contributions to several fundamental problems in number theory by himself and in collaboration with Terence Tao. Green's PhD thesis at Cambridge is a collection of several outstanding papers. In one of these papers he solved the Cameron- Erdős conjecture, which is a bound on the number of sum-free sets of positive integers up to a given number $N$. Green had also established an important result that any set of primes with relative positive density would contain infinitely many arithmetic progessions of length 3. It was this paper of Green in the 2005 Annals of Mathematics that caught the attention of Tao and which led to their collaborative and definitive result on arbitrarily long arithmetic progressions among the primes. Subsequently Green and Tao also collaborated in extending the Hardy-Ramanujan-Littlewood Circle Method by bringing in methods from ergodic theory.

In 2005 Green was appointed Hershel Smith Professor at Cambridge University. He was elected Fellow of the Royal Society in 2010 and was awarded the Sylvester Medal in 2014. He is currently Waynflete Professor of Pure Mathematics at Oxford University.

The 2008 Prize: Akshay Venkatesh of Stanford University was creating waves in the mathematical world by making powerful contributions to diverse areas, by himself and with a host of collaborators. His 2006 paper with H. Helfgott contained striking and original ideas, and provided the first non-trivial upper bound for the 3-torsion in class groups of quadratic fields. His joint work with Jordan Ellen-

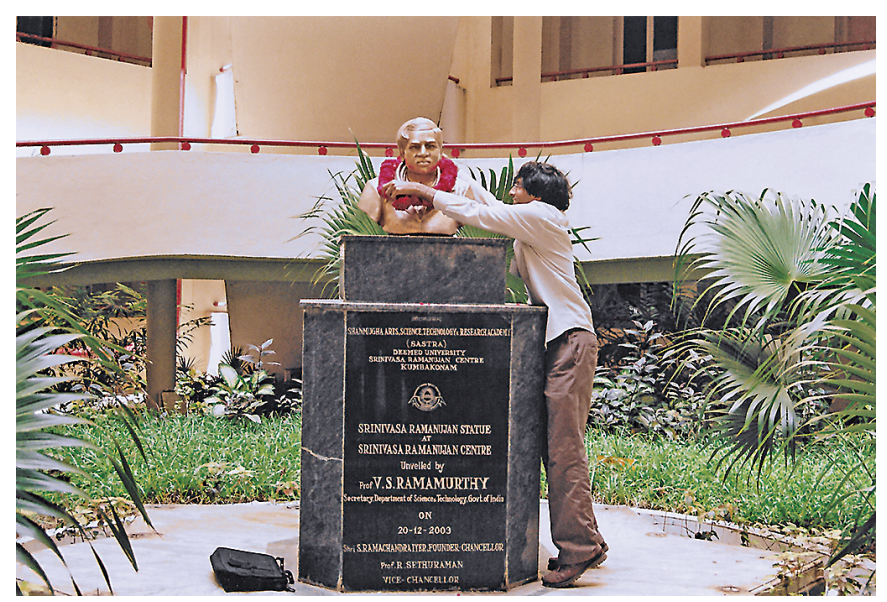

Figure 5. Akshay Venkatesh (Stanford), 2008 SASTRA PrizeWinner, garlanding the statue of Ramanujan at SASTRA University. 
berg on representing integral quadratic forms by quadratic forms had its roots in the work of Ramanujan.

An important and difficult problem in number theory is to asymptotically count number fields according to their discriminant. The case up to degree 5 had been solved by Manjul Bhargava. For large degrees, Ellenberg and Venkatesh provided the first major improvement over bounds in earlier work of Wolfgang Schmidt and thus broke an impasse of thirty years. Also of great importance was Venkatesh's work on sub-convexity of automorphic $L$-functions, and his joint work with E. Lindenstrauss, which settled a famous conjecture of Peter Sarnak concerning locally symmetric spaces.

After the SASTRA Ramanujan Prize, he has been awarded the Infosys Prize in 2016 and the Ostrowski Prize in 2017. In 2018 he was awarded the Fields Medal and appointed to the permanent faculty at the Institute for Advanced Study, Princeton.

The 2009 Prize: The 2009 Prize was awarded to Kathrin Bringmann for work related to Ramanujan's mock theta functions. A good portion of Ramanujan's Lost Notebook, namely, the loose sheets of paper on which he wrote hundreds of formulas shortly before he died in 1920, is devoted to mock theta functions. These are objects that are like the classical theta functions in their shape, but are not modular forms, yet their coefficients can be calculated with a degree of precision comparable to what can be done for functions that can be expressed in terms of theta functions. No one knew the exact relationship between theta functions and mock theta functions, and indeed determining this relationship was one of the tantalizing puzzles of mathematics. Following a lead provided by Sander Zwegers, Kathrin Bringmann and her post-doctoral mentor Ken Ono provided the key to unlock this mystery by showing how mock theta functions are intimately connected with harmonic Maass forms.

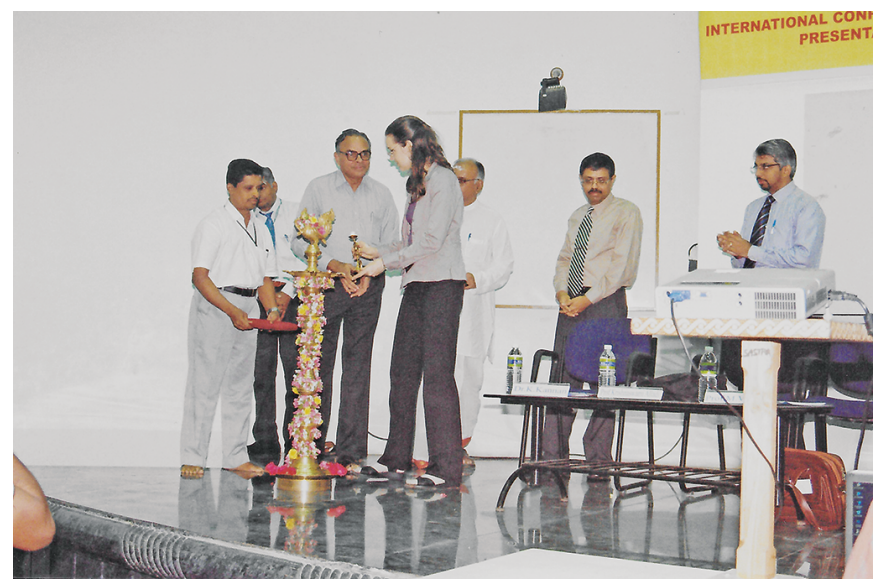

Figure 6. 2009 SASTRA Prize Winner Kathrin Bringmann (University of Cologne) lighting the ceremonial lamp to mark the opening of the Ramanujan Conference.
The 2010 Prize: As a PhD student at Columbia University, Wei Zhang had made seminal contributions by himself and in collaboration with others to a broad range of areas in mathematics including number theory, automorphic forms, trace formulas, $L$-functions, representation theory, and algebraic geometry.

In 1997 Steve Kudla constructed a family of cycles on Shimura varieties, and conjectured that their generating functions are actually Siegel modular forms. The proof of the Kudla conjecture for cycles of codimension 1 is a major theorem of Fields Medalist Richard Borcherds. In his PhD thesis, Zhang established conditionally, among other

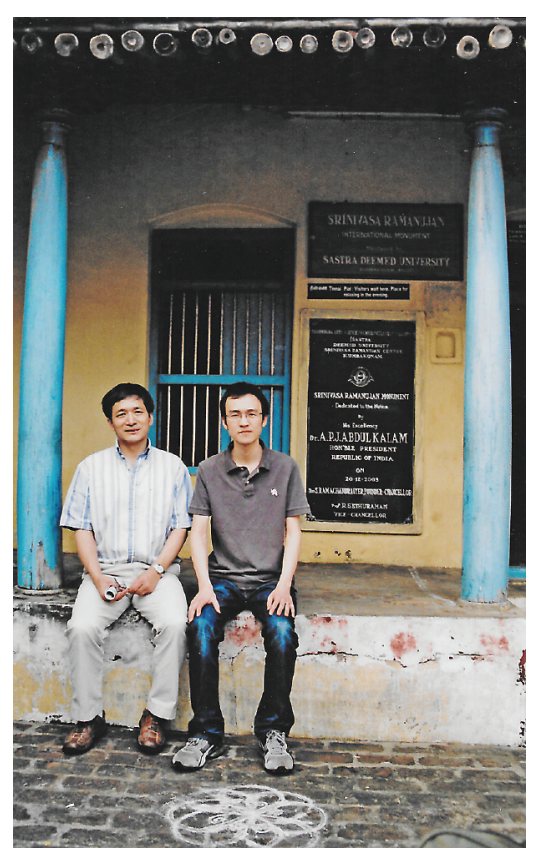

Figure 7. 2010 SASTRA Prize

WinnerWei Zhang (then at Harvard) seated (on the right) along with his former PhD advisor Shouwu Zhang (Columbia) at the entrance to Ramanujan's home in Kumbakonam. things, a generalization of Borcherds' result for higher dimensions and in that process essentially settled the Kudla conjecture. Zhang's thesis opened up major lines of research and led to significant collaboration with Xinyi Yuan and Shouwu Zhang. The three established an arithmetic analogue of a theorem of Waldspurger that connects integral periods to values of $L$-functions. In addition, Wei Zhang by himself had done outstanding work on relative trace formulas, and on Shimura varieties.

When Zhang received the SASTRA Prize, he was Benjamin Pierce Instructor at Harvard. In 2017 he was awarded the New Horizons Prize in Mathematics. He is currently a full professor at Columbia University.

The 2011 Prize: Roman Holowinsky of Ohio State University had made significant contributions at the interface of analytic number theory and the theory of modular forms. Along with Kannan Soundararajan he solved an important case of the famous Quantum Unique Ergodicity (QUE) Conjecture. This was a spectacular achievement.

In 1991, Zeev Rudnick and Peter Sarnak formulated the QUE Conjecture, which in its general form concerns the correspondence principle for quantizations of chaotic systems. One aspect of the problem is to understand how 
waves are influenced by the geometry of their enclosure. Rudnick and Sarnak conjectured that for sufficiently chaotic systems, if the surface has negative curvature, then the high frequency quantum wave functions are uniformly distributed within the domain. The modular domain in number theory is one of the most important examples, and for this case, Holowinsky and Soundararajan solved the holomorphic QUE conjecture.

The manner in which this solution came about is amazing. By a study of Hecke eigenvalues and an ingenious application of the sieve, Holowinsky obtained critical estimates for shifted convolution sums and this almost settled the holomorphic QUE conjecture for the modular domain except in certain cases where the corresponding $L$-functions behave abnormally. Simultaneously, Soundararajan approached the problem from an entirely different direction, was able to confirm the conjecture in several cases, and noticed that the exceptional cases not fitting Holowinsky's approach were covered by his techniques. Thus by combining the approaches of Holowinsky and Soundararajan, the holomorphic QUE Conjecture was fully resolved in the modular case.

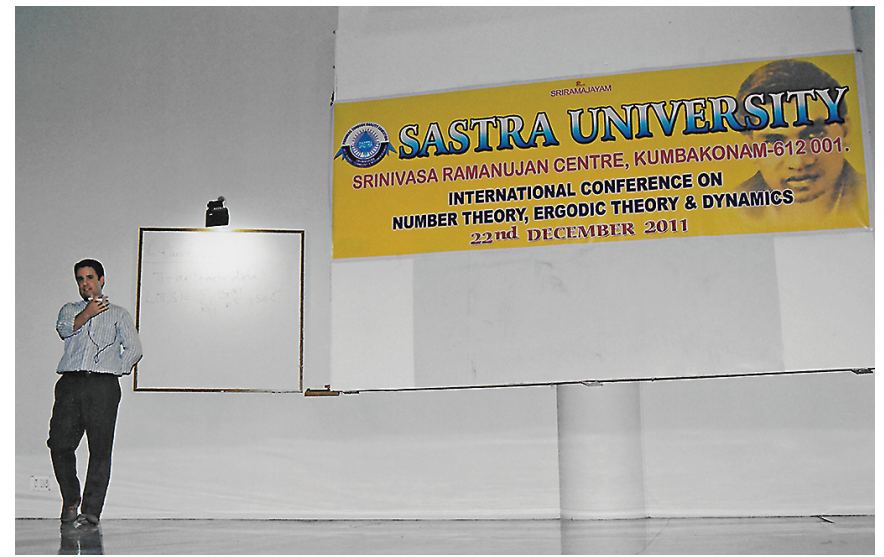

Figure 8. 2011 SASTRA Prize Winner Roman

Holowinsky (Ohio State) delivering the Ramanujan

Commemoration Lecture on December 22,

Ramanujan's birthday.

The 2012 Prize: Zhiwei Yun of Stanford University had made fundamental contributions to areas that lie at the interface of representation theory, algebraic geometry, and number theory. Yun's PhD thesis on global Springer theory at Princeton University opened up new vistas in the Langlands program. Springer theory is the study of Weyl group actions on the cohomology of certain subvarieties of the flag manifold called Springer fibers. Yun's global Springer theory deals with Hitchin fibers instead of Springer fibers, which he used to determine the actions of affine Weyl groups on cohomology.

Bao-Châu Ngô was awarded the 2010 Fields Medal for his proof of the Fundamental Lemma in the Langlands
Program. Yun made a breakthrough in the study of the Fundamental Lemma formulated by Jacquet and Rallis in their program of proving the Gross-Prasad conjecture on relative trace formulas. Yun's understanding of Hitchin fibrations enabled him to reduce the Jacquet-Rallis fundamental lemma to a cohomological property of the Hitchin fibration. In addition, Yun's work on the uniform construction of motives with exceptional Galois groups is considered to be very fundamental.

Since 2012 was the 125th Birth Anniversary of Ramanujan, Yun was awarded the SASTRA Prize in India's capital New Delhi, at a conference organized by the National Board for Higher Mathematics (of India), and co-sponsored by SASTRA University and Delhi University. That was the only year when this prize was not awarded in Kumbakonam.

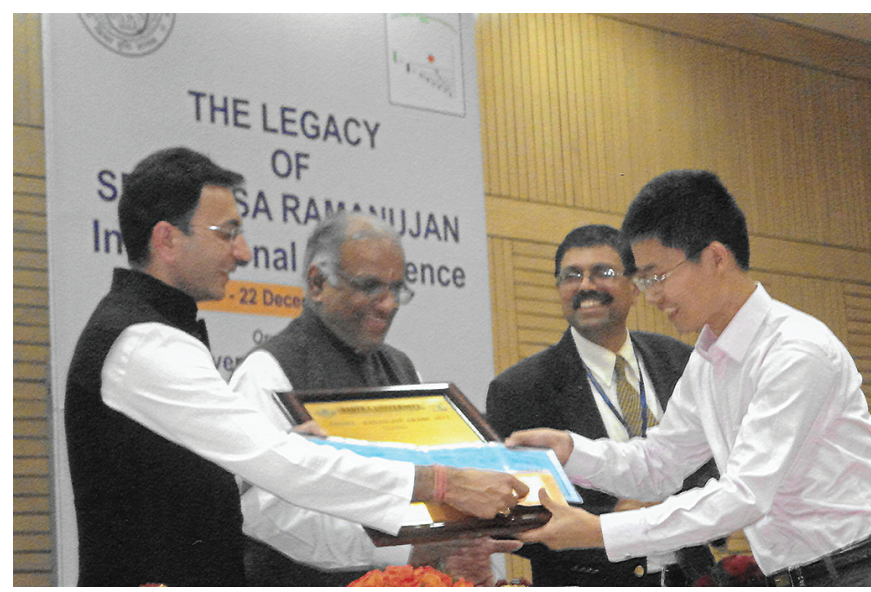

Figure 9. Zhiwei Yun (MIT and Stanford) receiving the 2012 SASTRA Ramanujan Prize from Minister of State Jitin Prasada at the University of Delhi on December 22. SASTRA Vice-Chancellor Sethuraman and Krishna Alladi are looking on.

When Yun received the SASTRA Prize, he had just been appointed to the permanent faculty at Stanford University after having completed his term as Moore Instructor at MIT. In 2018 he was a recipient of the New Horizons in Mathematics Prize.

The 2013 Prize: Peter Scholze of the University of Bonn had made revolutionary contributions to arithmetic algebraic geometry and the theory of automorphic forms. Already in his Masters thesis at Bonn, Scholze gave a new proof of the Local Langlands Conjecture for general linear groups based on a novel approach to calculate the zeta function of certain algebraic varieties. While this was groundbreaking, his $\mathrm{PhD}$ thesis at Bonn was a much bigger breakthrough: he developed a new $p$-adic machine called perfectoid spaces and used it brilliantly to prove a significant part of the weighted monodromy conjecture due to Deligne, thereby breaking an impasse of more than 30 years. Scholze extended his theory of perfectoid spaces 


\section{COMMUNICATION}

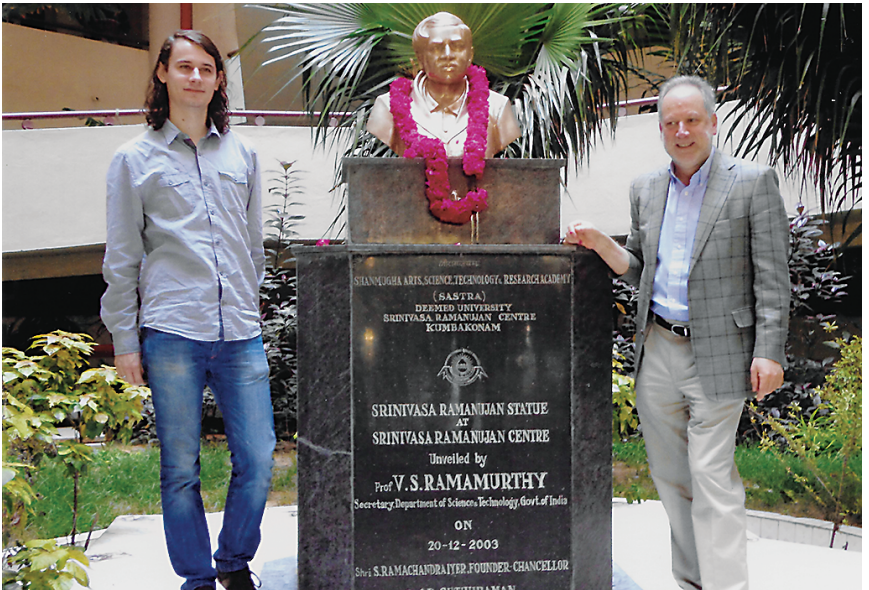

Figure 10. ( $L$ to R) 2013 SASTRA Prize Winner Peter Scholze (University of Bonn) and his former PhD Advisor Michael Rappoport (Bonn) standing next to the statue of Ramanujan at SASTRA University.

to develop a Hodge theory for rigid analytic spaces over $p$-adic ground fields, generalizing a theory due to Faltings for algebraic varieties.

Scholze was the youngest winner of the SASTRA Prize at the age of 25. Following that, he has been recognized with the AMS Cole Prize (2015), the Fermat Prize (2015), the Ostrowski Prize (2015), the Leibniz Prize (2016), and the Fields Medal (2018).

The 2014 Prize: James Maynard of Oxford University, England, and of the University of Montreal, had made outstanding contributions to some of the most famous problems on prime numbers. He obtained the strongest results at the time on the celebrated prime twins conjecture by showing that the gap between consecutive primes does not exceed 600 infinitely often. Not only did he significantly improve the path-breaking work of Goldston, Pintz, Ylldırım, and Zhang, but he achieved it with ingenious methods that were simpler than those used by others.

A generalization of the prime twins conjecture is the prime $k$-tuples conjecture, which states that an admissible collection of $k$ linear functions will simultaneously take $k$ prime values infinitely often. In the last one hundred years, several partial results towards the $k$-tuples conjecture were obtained by replacing prime values with "almost primes," namely numbers with a bounded number of prime factors. Another major achievement of Maynard was to significantly improve on the work of earlier researchers on $k$-tuples of almost primes.

Just before receiving the SASTRA Prize, Maynard announced the solution to the famous $\$ 10,000$ problem of Erdős on large gaps between primes. This was simultaneously announced by Kevin Ford, Ben Green, Sergei Konyagin, and Terence Tao, but Maynard's method was different and simpler. Maynard's results and methods have led to a resurgence of activity worldwide in prime number theory.

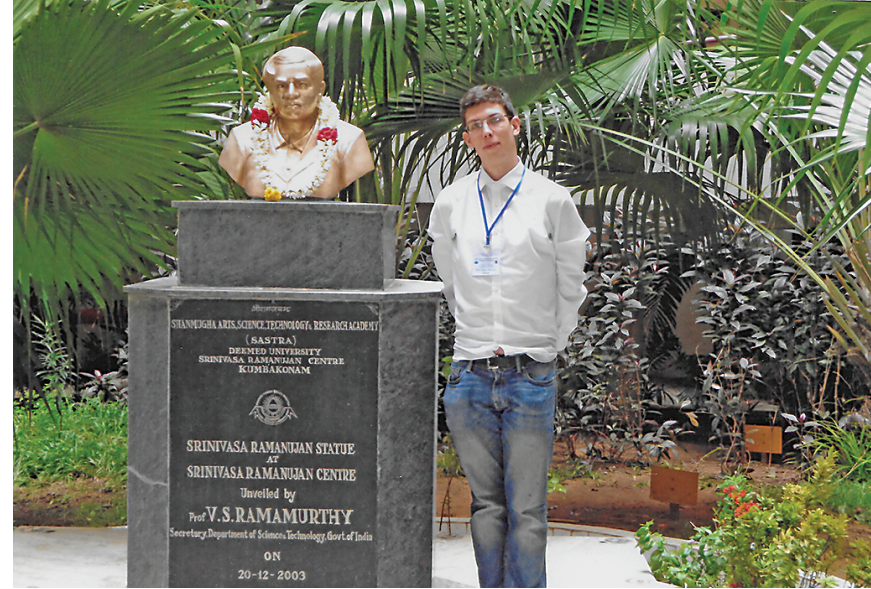

Figure 11. James Maynard (Oxford University), the 2014 SASTRA Prize Winner, standing next to the Ramanujan statue at SASTRA University.

After the SASTRA Prize, he received the Whitehead Prize in 2015 and the EMS Prize in 2016. He is currently a professor at Magdalen College, Oxford University.

The 2015 Prize: Jacob Tsimerman of the University of Toronto had made deep and original contributions to diverse parts of number theory, most notably to the André-Oort Conjecture, which states that special subsets of Shimura varieties that are obtained as Zariski closures of special points are finite unions of Shimura varieties. Shimura varieties are special algebraic varieties (such as moduli spaces of abelian varieties) that arise as quotients of suitable complex domains by arithmetic groups. Yves André initially stated this conjecture for one-dimensional sub-varieties, and subsequently Frans Oort proposed that

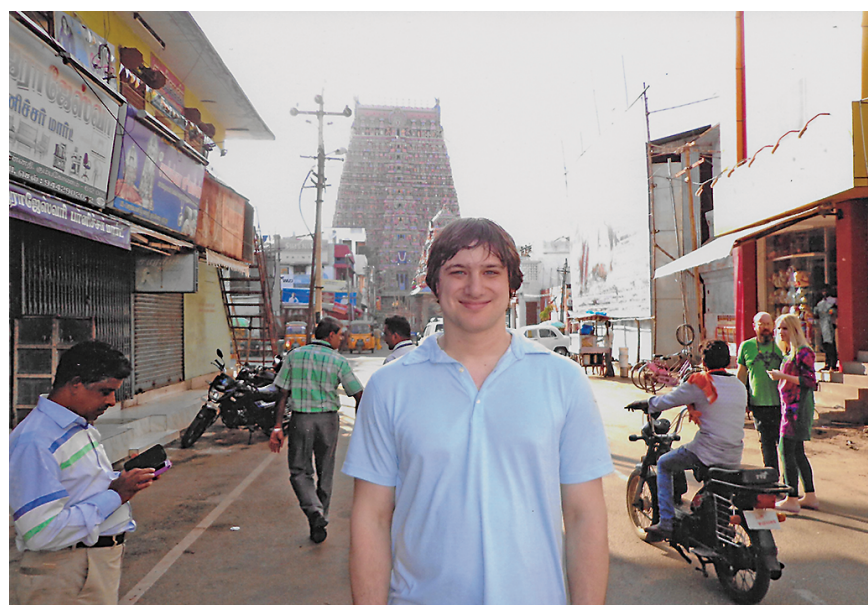

Figure 12. 2015 SASTRA Prize Winner Jacob

Tsimerman (University of Toronto) standing outside Ramanujan's home. The majestic Gopuram (entrance tower) of the Sarangapani Temple, where Ramanujan and family regularly worshipped, is seen in the background. 
it should hold more generally. A major achievement of Tsimerman in his Princeton PhD thesis of 2011 was to obtain certain unconditional bounds up to dimension 6 . Another important result in his thesis was to answer in the affirmative a question of Nick Katz and Oort whether there exists an abelian variety over the set of all algebraic numbers that is not isogenous to the Jacobian of a stable algebraic curve over the algebraic numbers.

Just before receiving the SASTRA Prize, Tsimerman gave a proof of the André-Oort Conjecture for the moduli spaces of principally polarized abelian varieties of any dimension-a result that was sought for a long time.

The 2016 Prize: This was shared by Kaisa Matomäki of the University of Turku, Finland, and Maksym Radziwill of McGill University, Canada, and Rutgers University. Their revolutionary collaborative work on multiplicative functions in short intervals shocked the mathematical community by going well beyond what could be proved

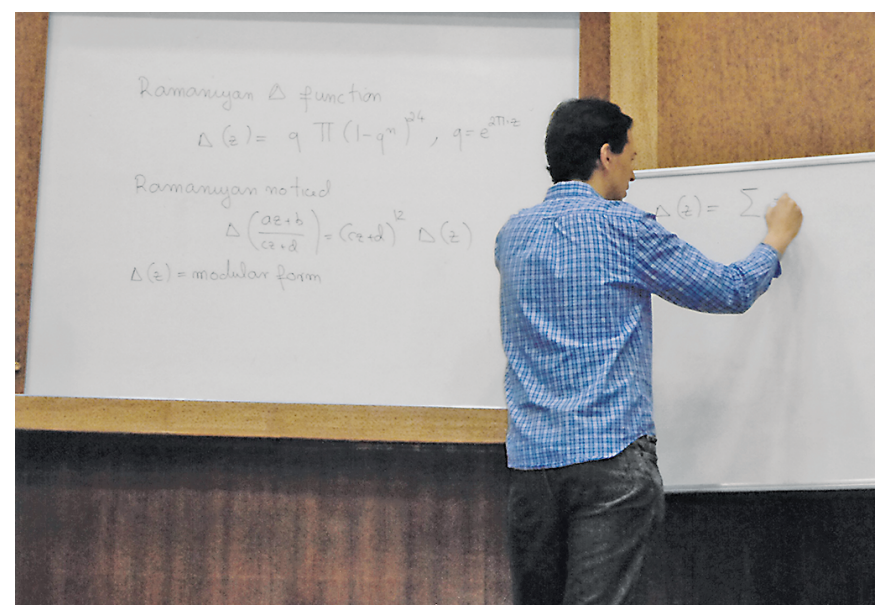

Figure 13. Maksym Radziwill (McGill University) giving the Ramanujan Commemmoration Lecture on his joint work with Kaisa Matomäki with whom he shared the 2016 SASTRA Prize.

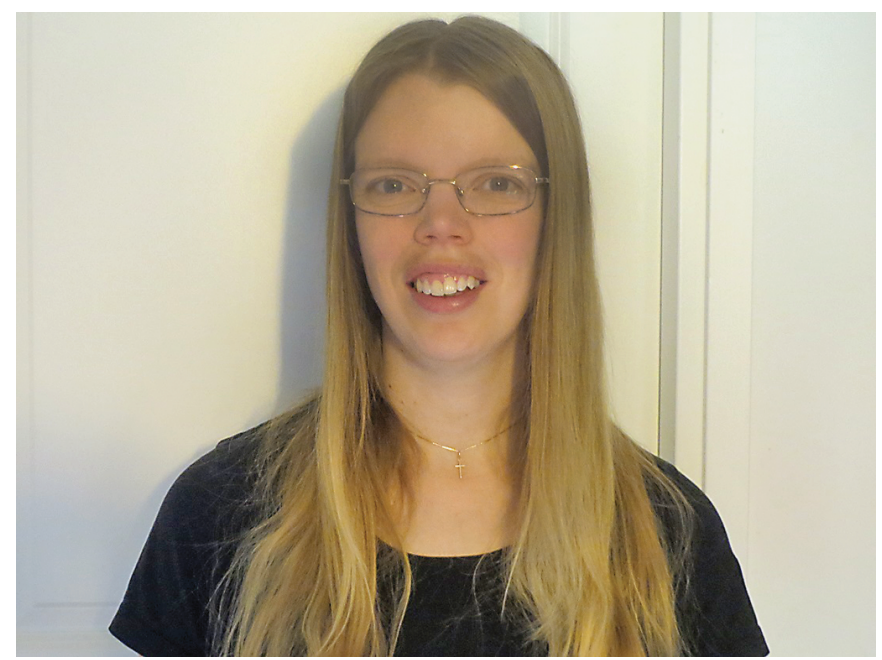

Figure 14. Kaisa Matomäki. previously even assuming the Riemann Hypothesis, and opened the door for a series of breakthroughs on some notoriously difficult questions such as the Erdős discrepancy problem and Chowla's conjecture, previously believed to be well beyond reach. Their stunning work on multiplicative functions in short intervals is best illustrated in terms of the Liouville lambda function, which takes value 1 when an integer has an even number of prime factors (counted with multiplicity), and value -1 at an integer with an odd number of prime factors. The statement that the lambda function takes values 1 and - 1 with asymptotically equal frequency is equivalent to the Prime Number Theorem; more refined statements on the relative error in this equal frequency are related to the Riemann Hypothesis. Such equal distribution results were known also for short intervals, namely intervals $[x, x+h]$, where $h$ is a fractional power $(<1)$ of $x$. The Riemann Hypothesis implies that powers of $h$ larger than $1 / 2$ will work. Instead of every interval of length $h$, if we require only "almost all" intervals of length $h$, it was known that $h$ could be made as small as the $1 / 6$ th power of $x$, and as small as a power of $\log x$ by assuming the Riemann Hypothesis. Matomäki and Radziwill shocked the world by showing unconditionally that equal frequency holds almost always as long as $h$ tends to infinity with $x$.

Sarvadaman Chowla conjectured that if any $k$ collection of values of 1 and -1 are given in any order, then the lambda function will take that sequence of values at $k$ consecutive integers with asymptotic frequency $1 / 2^{k}$. This conjecture is yet unsolved. In 2016, Matomäki, Radziwill, and Terence Tao proved that when $k=3$, each of the eight sign choices occur with positive proportion.

The 2017 Prize: Maryna Viazovska of the Swiss Federal Institute of Technology, Lausanne, was awarded the 2017 SASTRA Prize for her stunning solution in dimension 8 of the sphere-packing problem, and for her equally impressive joint work with Henry Cohn, Abhinav Kumar, Stephen D. Miller, and Danylo Radchenko, resolving the sphere-packing problem in dimension 24 , by building upon her fundamental ideas in dimension 8.

The sphere-packing problem has a long and illustrious history. Johannes Kepler asked for the optimal way to stack cannon balls (of uniform radius) and conjectured a configuration, but could not prove it. This Kepler Conjecture was resolved by Thomas Hales in 1998 by combining ingenious geometric optimization arguments with machine calculations. The sphere-packing problem in higher dimensions remained open.

In dimension 8 there is $E_{8}$, an exceptional Lie group with a root lattice of rank 8 , and in dimension 24 there is the Leech lattice. This gave some hope that the sphere-packing problem could be solved in dimensions 8 and 24. Noam Elkies and Henry Cohn made significant progress and conjectured the existence of certain magic auxiliary functions in dimensions 8 and 16, which, if determined, would resolve 
the conjecture in these dimensions. Viazovska produced these functions in dimension 8 by ingenious use of modular forms. Her proof is remarkably simple. Within a span of week, working at a furious pace, by extending the ideas in dimension 8, the sphere-packing problem in dimension 24 was resolved by Cohn, Kumar, Miller, Radchenko, and Viazovska.

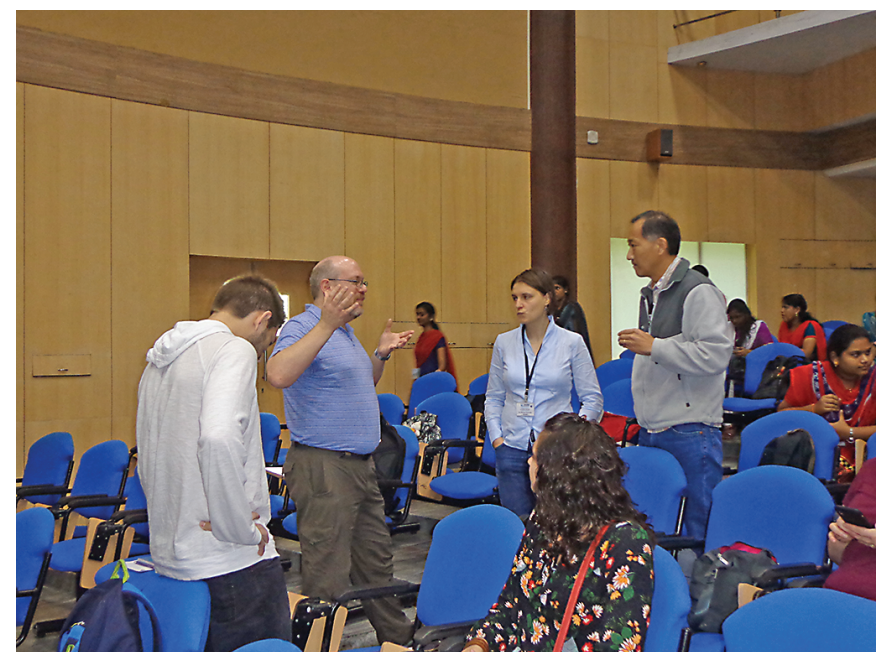

Figure 15. (L to R) Henry Cohn (Microsoft Research) discussing with 2017 SASTRA PrizeWinner Maryna Viazovska (EPF Lausanne) and Ken Ono (Emory)

In 2018 Viazovska was also awarded the New Horizons in Mathematics Prize.

Shaping the development of mathematics: The Fields Medals, with an age limit of 40 for the winners, were instituted with two lofty goals in mind: (i) to recognize pioneering work by brilliant young mathematicians, and (ii) to encourage these young researchers to continue to influence the growth of mathematics. The Fields Medalists have lived up to these great expectations. The SASTRA Ramanujan Prize has a more stringent age limit of 32, and so has recognized brilliant mathematicians even earlier in their careers. It is no exaggeration to say that the winners of the SASTRA Ramanujan Prizes have also shaped the development of mainstream mathematics and will continue to do so in the years ahead; the fact that the SASTRA laureates have subsequently won major prizes is a testimony to this. Indeed, two of the four 2018 Fields Medalists are former SASTRA Ramanujan Prize Winners: Akshay Venkatesh and Peter Scholze! Similarly, three of the four 2018 New Horizons in Mathematics Prize winners are former SASTRA awardees: Wei Zhang, Zhiwei Yun, and Maryna Viazovska.

\section{References}

1) Alladi K. Ramanujan's place in the world of mathematics essays providing a comparative study, Springer, New Delhi (2012), 177 pp.

2) Alladi K, "Ramanujan's thriving legacy," in Srinivasa Ramanujan going strong at 125, Parts I and II, Notices Amer. Math. Soc., I: 59 (2012), 1522-1537; II: 60 (2013), 10-22. MR3027107 and MR3052461

3) Ono K,. Honoring a gift from Kumbakonam, Notices Amer. Math. Soc., June-July 2006, 641-651. MR2235326

\section{Credits}

Figure 9 is courtesy of SASTRA.

Figure 14 is by Pekka Matomäki.

All other article photos and author photo are courtesy of the Krishnaswami Alladi.

The 2018 SASTRA Ramanujan Prize is shared by Yifeng Liu (Yale University) and Jack Thorne (Cambridge University) for spectacular contributions to algebraic geometry, automorphic representations, and number theory.

See p.113 of this issue for the Math People announcement about the 2018 SASTRA Prize to Liu and Thorne. 